\title{
PRIMERA GESTACIÓN LOGRADA A PARTIR DE ÓVULOS HUMANOS VITRIFICADOS
}

FIRST PREGNANCY ACHIIEVED FROM HUIMAN VITRIIFIED OOCYTES

Elkin Lucena, M.D, BMR*, Diana Patricia Bernal, MV, BMR**, Carolina Lucena, BS, $B M R^{* * *}$, Alejandro Rojas, M.D. ${ }^{\dagger}, B M R$, Sandra Mojica, BS, BMR ${ }^{\dagger \dagger}$, Ángela María Saa, BS, BMR ${ }^{\dagger \dagger}$, Zulma Suárez, BS, BMR ${ }^{\S}$, Abby Morán, Ps, BMR ${ }^{\S \S}$.

Recibido: septiembre 16/2004 - Revisado: septiembre 29/2004 - Aceptado: septiembre 30/2004

\section{RESUMEN}

La vitrificación de óvulos humanos tiene múltiples aplicaciones y demostró ser altamente efectiva en el manejo de una paciente con síndrome de hiperestimulación ovárica que se encontraba en tratamiento para infertilidad secundaria.

Objetivo: mostrar la eficiencia de la vitrificación de oocitos aplicada a óvulos en metafase II (MII) de una mujer con síndrome de hiperestimulación ovárica.

Procedimiento: se vitrificaron óvulos MII de una paciente con síndrome de hiperestimulación ovárica, para lo cual se utilizó la técnica cryotop y como agentes crioprotectores etilenglicol, dimetilsulfóxido (DMSO) y sacarosa. Dos meses después, una vez restablecida a la normalidad la condición clínica de la paciente, se desvitrificaron los óvulos, se utilizó la técnica de inyección intracitoplasmática de espermatozoides (ICSI) y 24 horas más tarde se evaluó fertilización, los cigotos obtenidos se cultivaron in vitro por 72 horas hasta el momento de la transferencia embrionaria intrauterina.

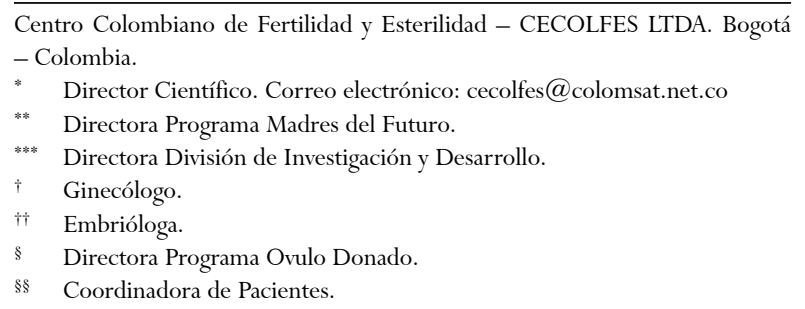

Resultados: la técnica de vitrificación con cryotop demostró ser una herramienta efectiva para preservar óvulos en lugar de embriones, además de disminuir los problemas éticos, morales, legales y religiosos que ésta conlleva.

Conclusiones: la vitrificación por cryotop proporciona altas tasas de supervivencia, fertilización, desarrollo embrionario y embarazo; así mismo, ofrece una nueva alternativa para el manejo del síndrome de hiperestimulación ovárica.

Palabras clave: oocito, vitrificación, supervivencia, viabilidad, gestación, ICSI, criopreservación, criobanco.

\section{SUIMMARY}

Among its multiple applications, vitrification of human oocytes has demonstrated to be highly effective in the handling of a patient with OHSS, who was under treatment for secondary infertility.

Objective: to demonstrate the efficiency of oocyte vitrification applied to metaphase II oocytes (MII), of women presenting OHSS.

Procedure: MII oocytes from a patient with OHSS were vitrified, using the cryotop technique with ethylenglycol, dimethylsulfoxide, and sucrose as cryoprotectants agents. Two months after, once the clinical stage of the patient was normalized, the oocytes were thawed and underwent ICSI; 24 hours later, fertilization was evaluated and zygotes were 
maintained under in vitro culture conditions during 72 hours, time of intrauterine embryo transfer.

Results: vitrification technique with cryotop demonstrated to be an effective tool for preservation of oocytes instead of freezing embryos due to all the ethical, moral, legal and religious points of view concerning preservation.

Conclusions: vitrification with cryotop provides high rates of survival, fertilization, embryo development and ongoing normal pregnancies, offering a new alternative for handling OHSS.

Key words: oocyte, vitrification, survival, viability, pregnancy, ICSI, cryopreservation, cryobank.

\section{INTRODUCCIÓN}

El oocito humano por sus condiciones estructurales y fisiológicas normalmente se ve afectado por las bajas temperaturas. Dentro de los principales inconvenientes o afecciones que sufren los óvulos durante la criopreservación encontramos:

1. Daño de la zona pelúcida. Estudios preliminares en ratones permitieron comprobar que la criopreservación inducía cambios en la zona pelúcida y/o liberación prematura del contenido de los gránulos corticales, ${ }^{1,2}$ con inhibición de la fertilización; dentro de los daños más importantes observados en la zona pelúcida por enfriamiento están las fracturas, disrupción de la membrana plasmática y desorganización extensiva del ooplasma, así como endurecimiento de la zona pelúcida, que es superado con el uso del ICSI.

2. Daño del citoesqueleto. Donde el eje meiótico ha demostrado ser altamente sensible a cambios de temperatura, en especial al enfriamiento; las anormalidades producidas pueden ser: reducción del tamaño del eje, desorganización y/o desaparición, algunas veces asociado con dispersión de los cromosomas. Sin embargo, dado que el dimetil sulfóxido (DMSO) favorece la polimerización de la tubulina mientras que las bajas temperaturas causan despolimerización de la misma, la combinación de DMSO y bajas temperaturas tiene un efecto protector. ${ }^{3-6}$

La criopreservación del óvulo humano es una herramienta útil en técnicas de reproducción asistida, tanto para las pacientes, como para las clínicas de infertilidad. El óvulo humano a pesar de ser una célula tan importante en la historia natural, ha sido muy esquiva a las técnicas de criopreservación.

Con la criopreservación de óvulos por curva lenta, utilizada desde 1986 cuando se consiguió la primera gestación a partir de óvulos congelados, ${ }^{7}$ en sus diferentes variaciones se emplean bajas tasas de enfriamiento $\left(<1^{\circ} \mathrm{C} / \mathrm{min}\right)$ y concentraciones de crioprotectores de aproximadamente $1,5 \mathrm{~mol} / \mathrm{Lit}$, donde la principal limitante es la toxicidad de los crioprotectores, daños por enfriamiento y formación de cristales de hielo intracelulares con tasas de embarazo, supervivencia y clivaje de $28 \%, 5 \%$, $76 \%$ y $60 \%$ respectivamente. ${ }^{2,7}$ La técnica no fue adoptada en forma amplia por la pobre reproducibilidad de sus resultados, ${ }^{2}$ motivo por el cual las clínicas de reproducción asistida no habían asumido la criopreservación de óvulos como una herramienta rutinaria en el manejo de pacientes con disfunción reproductiva. ${ }^{8}$

Mas adelante en 1997 se reporta el primer nacimiento de un embrión logrado a partir de óvulo descongelado e ICSI con una tasa de supervivencia del 33,3\%, tasa de fertilización del 50\%, tasa de clivaje del 50\%, donde realizaron, transferencia de un único embrión, logrando un embarazo. ${ }^{8}$ En otro estudio con 10 óvulos donados criopreservados, obtuvieron una tasa de supervivencia del 30\%, tasa de fertilización del 66,6\%, y una gestación. ${ }^{9}$

La vitrificación es un método de criopreservación en el cual hay solidificación de una solución cuando se enfría a velocidades altas formando un estado vidrioso, no por cristalización sino por elevación extrema de la velocidad; ${ }^{10}$ es un procedimiento simple que requiere menos tiempo de procesamiento y ha demostrado ser más seguro y efectivo que la criopreservación por curva lenta. ${ }^{11}$ 
Kuwayama, ${ }^{12}$ en el 2001, realizó modificaciones exitosas a la técnica de vitrificación, donde además de las altas tasas de enfriamiento y calentamiento se disminuyen las concentraciones de crioprotectores en un Minimum Volume Cooling (MVC). 5,6,12

Esta técnica busca minimizar o eludir los inconvenientes de la congelación por curva lenta como la formación de cristales de hielo intra y extracelulares, lesiones por enfriamiento, toxicidad de los crioprotectores, choque osmótico, lo cual ha sido demostrado con altas tasas de supervivencia y finalmente altas tasas de embarazo. ${ }^{5,12}$

La técnica de vitrificación por cryotop hasta el momento garantiza la estabilidad del eje meiótico y los cromosomas, de forma que evita anormalidades genéticas asociadas a la técnica de criopreservación. ${ }^{12}$

La criopreservación es una herramienta que facilita el almacenamiento a largo plazo de oocitos de pacientes en peligro o riesgo de perder su función ovárica y permite una gran flexibilidad en los servicios de fertilidad, aliviando las preocupaciones éticas asociadas con la preservación de embriones. ${ }^{1}$

Aunque el síndrome de hiperestimulación ovárica $(\mathrm{SHO})$ es una complicación iatrogénica rara derivada de la hiperestimulación ovárica controlada (HOC), puede tener grandes repercusiones multisistémicas y algunas de ellas fatales. ${ }^{13}$ Dentro de las estrategias para la prevención de estas complicaciones desde 1990 se cuenta con la congelación de embriones, ${ }^{14}$ como lo aplicó el grupo de Wada en $1993,{ }^{15,16}$ criopreservando selectivamente todos los embriones de las mujeres que presentaban niveles mayores de $3.500 \mathrm{pg} / \mathrm{ml}$ en el día de aplicación de gonadotropina coriónica humana (hCG).

La realidad es que en la actualidad la criopreservación de oocitos es utilizada en un número limitado de clínicas particulares en Italia, Alemania, EEUU, Sur América y Asia. ${ }^{6,8,17,18}$

\section{MATERIALES Y MÉTODOS}

Una paciente de 21 años, con infertilidad secundaria por dos embarazos espontáneos ectópicos previos, por lo cual se le efectuó salpingectomía bilateral, se sometió a un esquema de HOC. Alcanzó niveles séricos de estradiol de $5.000 \mathrm{pg} / \mathrm{ml}$, motivo por el cual se acudió a la criopreservación de óvulos, con el propósito de evitar la severidad de la sintomatología. Dos meses después del restablecimiento de su condición clínica se procede a la desvitrificación y fertilización de estos óvulos para transferencia intrauterina y se consigue un embarazo normal.

\section{Hiperestimulación ovárica controlada}

Se consiguió con el uso del agonista GnRH a partir del primer día de la menstruación, con dosis de 0,5 mg/día vía subcutánea; a partir del tercer día del ciclo se inició estímulo con r-FSH a dosis de 150-250 UI/día hasta obtener folículos de 18-22 $\mathrm{mm}$ de diámetro mayor, momento en el cual se aplicaron 10.000 UI de hCG y 34-36 horas después bajo visión ecográfica se practicó la recuperación de los óvulos vía transvaginal, utilizando ecógrafo Hitachi (EUB-405) con transductor transvaginal de 6,5 MHz.

\section{Selección y vitrificación de óvulos en el laboratorio}

Los oocitos una vez recuperados fueron denudados con hialuronidasa $80 \mathrm{UI} / \mathrm{ml}$ (Lab Sage In vitro Fertilization Inc, Troumbull USA), se evalúo su madurez y se vitrificaron mediante la técnica cryotop, diseñada y descrita por Kuwayama y col.; ${ }^{1,10}$ la vitrificación se realizó con el método MVC, con soluciones crioprotectoras (Kitazato Co, Tokyo, Japan), los óvulos se equilibraron en solución ES: EG+DMSO (Equilibration solution - Kitazato Co, Tokyo, Japan), durante diez minutos y luego se colocaron en solución de vitrificación VS (Kitazato Co, Tokyo, Japan) consistente de EG + DMSO+ sacarosa; los óvulos se cargaron en forma rápida en MVC aprox $2 \mu \mathrm{l}$ de VS y se sumergieron directamente en nitrógeno líquido (NL), manejando tasas rápidas de enfriamiento $\left(-23.000^{\circ} \mathrm{C} / \mathrm{min}\right)$.

\section{Preparación endometrial}

Dos meses después de la aspiración transvaginal, la paciente se sometió a un esquema de preparación 
endometrial con valerianato de estradiol en dosis crecientes, inicialmente $2 \mathrm{mg}$ y en el día 14 del ciclo dosis de $6 \mathrm{mg}$ /día, manteniéndose así hasta el momento de la prueba de embarazo. A partir del día 13 (tres días antes de la transferencia de embriones), se inició la aplicación de $50 \mathrm{mg}$ de progesterona natural inyectable.

\section{Desvitrificación de óvulos y transferencia de embriones}

Los óvulos se desvitrificaron por medio de tasas rápidas de calentamiento $\left(12.000^{\circ} \mathrm{C} / \mathrm{min}\right)$, colocándolos un minuto en solución TS (Thawing solution) de desvitrificación, sacarosa $1 \mathrm{M}$ a $37^{\circ} \mathrm{C}$; luego se equilibraron lentamente en solución DS (Diluent solution Kitazato Co, Tokyo, Japan) durante tres minutos a temperatura ambiente y se realizaron dos lavados consecutivos en medio WS (Washing solution, Kitazato Co, Tokyo, Japan). Inmediatamente se colocaron en medio de fertilización en incubación durante dos a cuatro horas cuando se evaluó su viabilidad y a los viables se les realizó ICSI. Veinticuatro horas más tarde se evaluó la presencia de pronúcleos y se continuó el cultivo in vitro por 72 horas hasta el día de la transferencia intrauterina. A la paciente se le aplicó gel vaginal de progesterona cuatro horas antes del procedimiento que se llevó a cabo bajo guía ecográfica transabdominal con catéter soft frydman set echo ${ }^{\circledR}$ (Laboratoire CCD, París, Francia).

\section{RESULTADOS}

Después de la HOC se recuperaron once óvulos, nueve de ellos en metafase II; se vitrificaron siete, de los cuales al momento de la desvitrificación sobrevivieron cinco. A las 24 horas post ICSI cuatro de ellos presentaban pronúcleos y 72 horas después de cultivo se transfirieron tres embriones de seis blastómeras en el día 16 del ciclo de la paciente (tabla 1). Siete días después de la transferencia intrauterina se realizó seguimiento hormonal (tabla 2) hasta observar latido cardíaco. Cuando se realizó este escrito la paciente tenía una gestación normal de una niña de 20 semanas.

\section{DISCUSIÓN}

La criopreservación de óvulos humanos trae múltiples ventajas y beneficios tanto para las mujeres para salvaguardar su fertilidad y futura maternidad, como para las clínicas o centros de reproducción asistida, con lo cual pueden ofrecer nuevas y mejores alternativas a sus pacientes en tratamiento.

Tabla 1. Evolución de los óvulos postdesvitrificación

\begin{tabular}{lc} 
Evento & Número \\
Óvulos vitrificados & 9 \\
Óvulos desvitrificados & 9 \\
Óvulos recuperados & 7 \\
Óvulos viables & 5 \\
Óvulos fertilizados & 4 \\
Embriones transferidos & 3 \\
Gestación & + \\
\hline
\end{tabular}

\begin{tabular}{|c|c|c|c|c|c|c|c|}
\hline Día post T.E. & 7 & 12 & 16 & 23 & 30 & 38 & 45 \\
\hline P4 ng/dl & 15 & 20 & $>40$ & $>40$ & 17 & 23 & $>40$ \\
\hline Estradiol pg/ml & 133 & 220 & 379 & 207 & 459 & 1.670 & 23.850 \\
\hline $\mathrm{B}$ hCG mUI/ml & - & 176 & 945 & 12.147 & 53.600 & 113.201 & 185.200 \\
\hline
\end{tabular}


La posibilidad de vitrificar óvulos de pacientes con SHO conserva las ventajas de la criopreservación de embriones hasta el momento utilizada; además evita los dilemas éticos, morales, legales y de credo que conlleva la criopreservación de los mismos, conservando las tasas de fertilización, desarrollo embrionario y embarazo.

Dentro de las múltiples ventajas que ofrece la vitrificación de óvulos tenemos el que permite un adecuado manejo de programas de donación de óvulos, así como que constituye una opción frente a inconvenientes en el momento de la obtención de la muestra seminal, como método para salvaguardar la fertilidad de pacientes oncológicas y una alternativa para controlar el reloj biológico de la maternidad, entre otras.

De esta forma se presenta el primer embarazo logrado a partir de óvulos vitrificados en Colombia y Latinoamérica.

\section{REFERENCIAS}

1. Al-Hasani S, Diedrich K, Van der Ven, Reineke A, Harjte M, Krebs D. Cryopreservation of human oocytes. Hum Reprod 1987;2:695-700.

2. Gook D, Osborn S, Johnson W. Cryopreservation of mouse and human oocytes using 1,2 propanediol and the configuration of the meiotic spindle. Hum reprod 1993;8:1101-9.

3. Tucker M, Wright G, Morton P, Shangus L, Massey J, Kort H. Preliminary experience with human oocyte cryopreservation using 1,2 propanediol and sucrose. Hum Reprod 1996;11:1513-5.

4. Eroglu A, Toth TL, Toner M. Alterations of the cytoskeleton and polyploidy induced by cryopreservation of metaphase II mouse oocytes. Fertil Steril 1998; 69:944-57.

5. Katayama KP, Stehlik J, Kuwayama M, Kato O, Stehlik E. High survival rate of vitrified human oocytes results in clinical pregnancy. Fertil Steril 2003;80:223-4.

6. Kuleshova L, Gianaroli L, Magli C, Ferraretti A, Trounson A. Birth following vitrification of a small number of human oocytes: case report. Hum Reprod 1999;14:3077-9.
7. Chen C. Pregnancy after oocyte cryopreservation. Lancet 1986;1:884-6.

8. Porcu E, Fabbri R, Seracchioli R, Ciotti P, Magrini O, Flamigni C. Birth of healthy female after intracytoplasmic sperm injection of cryopreserved human oocytes. Fertil Steril 1997;68:724-6.

9. Polak de Fried E, Notrica J, Rubinstein M, Marazzi A, Gómez M. Pregnancy after human donor oocyte cryopreservation and thawing in association with intracytoplasmic sperm injection in a patient with ovarian failure. Fertil Steril 1998;69:555-7.

10. Shaw JM, Jones GM. Terminology associated with vitrification and other cryopreservation procedures for oocytes and embryos. Hum Reprod Update 2003;9:583-60.

11. Kuleshova LL, Lopata A. Vitrification can be more favourable than slow cooling Fertil Steril 2002;78:449-53.

12. Kuwayama M. Vitrification of human oocytes and embryos. In: Suzuki S (ed). IVF update [In Japanese]. Tokyo: Medical view co.; 2001. p. 230-34.

13. Mozes M, Bogokowsky H, Antebi E, Lunenfeld B, Rabau E, Serr DM, Dacid A, Salomy M. Thromboembolic phenomena after ovarian stimulation with human gonadotrophins. Lancet 1965;2:1213-5.

14. Amso NN, Ahuja KK, Morris N, Shaw RW. The management of predict ovarian hyperstimulation involving gonadotrophin-releasing hormone analog with elective cryopreservation of all pre-embryos. Fertil Steril 1990;53:1087-90.

15. Wada I, Matson Pl, Troup SA, Morrol DR, Hunt L, Liebermant BA. Does elective cryopreservation of all embryos from women at risk of ovarian hyperstimulation syndrome reduce the incidence of the condition. Br J Obstet Gynaecol 1993;100:265-9.

16.Aboulgar MH. Mansour RT. Ovarian hyperstimulation syndrome: classifications and critical analysis of preventive measures. Hum Reprod Update 2003;9:275-89.

17. Azambuja R, Stachecki J, Badalotti M, Michelon Petracco A. Nascimento de feto a termo após congelamento de oocitos. J Brasileiro Reprod Assist 2002;6: 119-21.

18. Van der Elst J. Oocyte freezing: here to stay? Hum Rep Update 2003;9:463-70. 